\title{
De Reclus a Harvey, la resignificación del territorio en la construcción de la sustentabilidad
}

\author{
From Reclus to Harvey, territory resignification \\ in the construction of sustainability
}

Arturo V. Arreola Muñoz*

Antonio Saldívar Moreno**

Resumen: el objetivo de este artículo es reflexionar teórica y conceptualmente sobre el territorio y su resignificación en la construcción de la sustentabilidad. Se hace referencia a la teoría de sistemas complejos, desde un enfoque constructivista, y de la geografía y la ecología política, como aproximación a las nociones de apropiación territorial, territorialización, desterritorialización y reterritorialización. La discusión deductiva de cinco tesis concluye con un postulado que retoma la propuesta de Gunderson y Holling (2002), para explicar los procesos adaptativos de los sistemas socioterritoriales, los cuales están mediados a partir de cadenas dialógicas. Actualmente, desde los movimientos sociales emergentes, se tejen innovaciones que recrean de manera híbrida conceptos como diversidad, autonomía, equidad y

* Investigador-consultor en temas ambientales, Instituto para el Desarrollo Sustentable en Mesoamérica A. C., El Colegio de la Frontera Sur/doctorado en ecología y desarrollo sustentable. Av. Cristóbal Colón \#35B, barrio El Cerrillo C.P. 29220, San Cristóbal de las Casas, Chiapas, México. Teléfono: (967) 678 2263. Correo electrónico: arturovam@yahoo.com.mx

** Investigador-profesor, grupo académico Procesos Culturales y Construcción Social de Alternativas del Departamento Sociedad y Cultura, El Colegio de la Frontera Sur. Carretera Panamericana s/n, esquina Periférico Sur, barrio Ma. Auxiliadora 29290, San Cristóbal de Las Casas, Chiapas, México. Teléfono: (967) 674 9000, extensión 1543. Correo electrónico: asaldivar@ecosur.mx 
sustentabilidad. Ello permite resignificar el diálogo político del paradigma por el territorio entre los espacios de esperanza -redes antisistémicas-, los exclusivos -redes globalizadas-y los de desesperanza -espacios fragmentados-.

Palabras clave: territorialización; movimientos socioterritoriales; sustentabilidad; ecología política; globalización; sistemas sociales.

Abstract: the purpose of this article is to theoretically and conceptually reflect on the territory and its resignification in the construction of sustainability. It refers to the complex systems theory from a constructivist approach, and to geography and political ecology as an approximation to the notions of territorial appropriation, territorialisation, deterritorialisation and reterritorialisation. Deductive discussion of five theses concludes with a postulate that takes up Gunderson and Holling's proposal (2002) so as to explain the adaptive processes of the socio-territorial systems, which are mediated through dialogic chains. Nowadays, the emerging social movements generate innovations that in a hybrid way recreate concepts such as diversity, autonomy, equity and sustainability. It enables to resignify the political dialogue of the paradigm through the territory among the spaces of hope (antisystemic networks), the exclusive ones (globalized networks) and the ones of despair (fragmented spaces).

Key words: territorialisation; socio-territorial movements; sustainability; political ecology; globalization; social systems.

\section{Introducción}

La relevancia reciente del discurso sobre el territorio tiene implicaciones en los campos ontológico, epistemológico e ideológico. La 
discusión se ha concentrado en explicar los sistemas sociales como constructores del territorio, lo que permite continuar el debate sobre la relación sociedad-naturaleza y sus implicaciones espaciales. La idea de que la humanidad ejerce un dominio sobre la naturaleza prevaleció en el pensamiento de los geógrafos racionalistas, quienes proponían una suerte de espacio natural preexistente, en donde las sociedades distribuyen objetos y procesos. Al compartir esta perspectiva objeto-objeto, los evolucionistas plantearon incluso que la naturaleza determinaba el devenir social. Reclus (1905) fue el primero en cuestionar estos planteamientos $y$, desde una ética anarquista, se centró en reconocer el lazo íntimo que reúne la sucesión de los hechos humanos y la acción de las "fuerzas telúricas". A partir de ahí se debate el abordaje conceptual sobre el territorio, al reconocer su sentido ontológico, y considerar que realmente existe (con un enfoque materialista o idealista) o una propuesta epistemológica, donde la noción interviene como un instrumento heurístico del que se vale el investigador para aproximarse a la realidad (Santos 2000).

La existencia humana y los procesos naturales comparten el espacio y representan la realidad tangible que se conoce. Los planteamientos teóricos, que se opusieron a los positivistas, proponían que su interrelación tenía un carácter sujeto-objeto, pues las sociedades trasformaban el medio físico circundante, y lo adaptaban para satisfacer sus necesidades; las configuraciones territoriales generadas eran el reflejo de las motivaciones humanas (Palacios 1983). Sin embargo, cuando la sociedad actúa sobre el espacio no lo hace sobre objetos considerados como realidad física, es decir, como naturaleza primigenia, sino sobre realidad social de formas-contenido, como funciones sociales ya valorizadas a las cuales se busca ofrecer o imponer un valor nuevo (Santos 2000). Desde esta perspectiva, la relación sociedad-naturaleza es un proceso que internaliza a la naturaleza en el sistema social, lo que produce que ambas se readecuen, readapten y modifiquen constantemente (Ramírez 2003). Esta referencia no sólo permite la "desnaturalización" de la naturaleza, lo cual da paso a la noción de ambiente, sino que también establece que esta relación tiene un carácter sujeto-sujeto, en donde el territorio es construido por un conjunto indisoluble, solidario y contradictorio de acciones y formas-contenido, que ocurren en un contexto histórico. Los sujetos y sus acciones, a lo largo del tiempo, constituyen el tema central de 
estudio de la investigación de lo territorial, que considera las prácticas sociales y los sentidos simbólicos en su relación con la naturaleza, por lo cual requiere un abordaje interdisciplinario (Llanos 2010). La noción de territorio implica relaciones verticales entre acciones y formas-contenido, por medio de la interacción entre escalas, niveles y tiempos; así mismo, incluye a las relaciones horizontales de carácter funcional, de interdependencia, de selección, de reproducción, de sustitución o de cambio, que son específicas para cada lugar.

La dinámica territorial está ligada a los cambios en las representaciones, procesos y articulaciones de los sistemas sociales. Esta semantización del espacio, propuesta por los geógrafos de la escuela humanista y crítica, resalta la apertura hacia una noción multidimensional y compleja de la realidad, que se deriva de la consideración de hibridez de aspectos culturales, políticos y económicos explorada por Santos (2000) y Haesbaert (2004); también, puede servir de referente para explicar la internalización de la naturaleza en la sociedad, y desechar la perspectiva analítica que supone que ambas funcionan mutuamente como externalidades.

El territorio se puede considerar como la manifestación "de una determinada configuración social no exenta de conflictos que involucran a una diversidad de actores que comparten el espacio" (Gómez y Hadad 2007, 8). Esta noción refleja que es un producto construido, resultado de un proceso interactivo a través del cual es interpretado y reinterpretado de forma compleja; al ser una representación, siempre es susceptible de ser trasformado. Se puede decir, parafraseando a Paul Watzlawick (1981), que territorio es lo que así es denominado por un número suficientemente grande de personas; en este sentido, es una convención interpersonal. Proponer una definición operacional de territorio, desde este enfoque, sugiere considerar dos aspectos que conjugan lo expuesto por Mallon (1995) y Zúñiga (1998); por un lado, el derecho que se atribuye un grupo social sobre un conjunto determinado de formas-contenido, concebido en términos legales o simbólicos y, por otro, las acciones que aluden a la lucha por el control de los procesos políticos, económicos, el uso del suelo, la conformación de unidades productivas, la organización e identidad grupal y la aplicación de normatividades de índole diversa (Guizar 2005). 
La construcción social del territorio no es excluyente de la individualidad, ni de la vida familiar, sino que parte del sistema de intereses no estructurados que tienen, de la percepción y concepción de necesidades y potencialidades para cubrirlas. Se construye cuando dichos propósitos se estructuran a partir de las propuestas valoradas por una colectividad, lo cual deriva en la generación de nociones dialógicas que funcionan como formas-contenido y acciones sociales. Guba (1990) denomina a este mecanismo el diálogo del paradigma, ${ }^{1}$ es el de la disputa o el consenso, en el que se pretende excluir o integrar al "otro", al poner en marcha estrategias para el control dialógico de los procesos, lo cual implica fijar una hegemonía. Este diálogo entre los intereses y los propósitos se concreta en una estructuración jerárquica de relaciones sociales, que construye una distribución territorial orientada a beneficiar al grupo mejor posicionado o hegemónico (Guizar 2005).

El territorio es, por tanto, el "constructo" de un proceso complejo que implica un dominio (económico-político) y una apropiación (simbólico-cultural) de formas-contenido asignadas por los sistemas sociales (Haesbaert 2004). Para Claude Raffestin (1993), las representaciones configuran la mediación que permite sustantivar el espacio como territorio, de esta manera: "La dimensión espacial constituye su condición y fundamento, pero como una abstracción que se concretiza a través de la acción de un actor sintagmático que es realizador de un programa. Entonces el espacio, una vez representado, ya no es espacio sino territorio resultado de una apropiación” (Gómez y Hadad 2007, 6). Desde la perspectiva de la geografía cultural, Fernández (2006) profundiza en estas ideas, y reconoce que la cultura es el medio a través del cual la gente trasforma el mundo material en uno de símbolos. Este viraje epistemológico de la geografía se deriva de las reformulaciones propias del giro interpretativo de las ciencias sociales.

1 El objetivo de este diálogo no es determinar cuál interés o propósito es el que va a "ganar"; por el contrario, permite acceder a otro nivel, en el que todos los paradigmas se sustituyen por uno emergente, el cual no se aproxima más a la "verdad”, como quisiera suponer un positivista, sino que crece epistemológicamente al ser considerado más informado y complejo (Guba 1990). 


\section{Discusión}

Primera tesis: la territorialización expresa la capacidad de autoorganización y respuesta al entorno de los sistemas sociales

La noción de territorio tiene que ver con los ciclos y los elementos materiales, bióticos y abióticos que le son intrínsecos de manera natural, a los cuales se les asignan formas-contenido, por medio de la acción cultural, que son representaciones dinámicas con diversos grados de complejidad objetiva y subjetiva. El territorio también es escenario económico, donde cada unidad tiene un peso específico independiente de las demás (Hiernaux 1999), por lo que adquiere un valor diferenciado al insertarse con otras dentro del proceso de acumulación de capital; su posición, en cuanto a escala, localización y emplazamiento, le confiere una dimensión espacial. De manera individual, gremial o colectiva, los grupos sociales luchan por lograr el control o hegemonía sobre el territorio, lo cual les otorga su estatus político, al ser un constructo en cambio constante, la noción de territorio implica necesariamente una perspectiva histórica.

La apropiación territorial se conceptualiza como el acto por el que una colectividad establece la ocupación y control de una porción del espacio para hacerlo suyo, con el fin de usufructuar y aprovechar sus recursos, definir las modalidades de acceso y organizar las actividades que le permitan satisfacer sus necesidades (Godelier 1989, citado en Márquez 2002). La construcción de un territorio comienza cuando un grupo social se apropia de un espacio, al darle contenido, dicho proceso se denomina territorialización (Sánchez 1998, citado en Monroy 2004). De acuerdo con Weber y Reveret (1993, citados en Del Carpio 2003), la apropiación del territorio tiene tres dimensiones, las cuales se constituyen en los mecanismos de la territorialización.

La dimensión subjetiva corresponde a las representaciones que el grupo social asigna y ejerce en el territorio que construye, las cuales conforman su identidad, sentido de adscripción, pertenencia y apego. Indica sistemas de valores (formas-contenido) y las relaciones entre éstos a propósito de las acciones culturales. Constituye el medio por el cual se reafirma el ser, derecho que reivindican todos los individuos y grupos humanos (Escobar 2005). 
La dimensión concreta alude a los usos que la población hace de los recursos naturales, se manifiesta en prácticas de referencia y en la organización del trabajo. Articula sistemas sociales y ambientales, por ello pueden ser significativas las aportaciones de las ciencias de la naturaleza al análisis de este mecanismo de apropiación. Incluye la forma en que se distribuyen los recursos, lo cual no está condicionado sólo por el mercado para la utilización óptima de los factores de producción o inversión, sino también por el sistema de representación.

La dimensión abstracta constituye las normas y las reglas que la colectividad establece para acceder a los recursos del territorio, su distribución y sistema de propiedad. Dicho acceso en general no es libre, está regulado por instituciones culturales, por derechos colectivos o históricos que a menudo entran en contradicción con las formas de tenencia o propiedad instituidas de manera concreta.

La operación de estas dimensiones, como mecanismos de territorialización, se puede abordar a partir del enfoque de los sistemas complejos propuesto por García (1986). El territorio es uno de ellos, y está compuesto por varias partes entrelazadas (dimensiones) cuyos vínculos contienen información propia y adicional a la inherente a cada elemento, por ello, para describirlo es necesario conocer el funcionamiento de las partes, y también cómo se relacionan entre sí. El conjunto de la información es superior a la contenida en la suma de las partes; se trata de la concepción holística, en donde el todo es más que la suma de las partes. Como resultado de las interacciones entre elementos surgen propiedades nuevas, denominadas emergentes, que no se pueden explicar a partir de los elementos precedentes (García 2000). Desde este punto de vista, por sí solas, las dimensiones de la apropiación territorial no pueden explicar la territorialización, que es una propiedad emergente, que representa el derecho a estar de cada grupo social (Escobar 2005).

El concepto de emergencia tiene una relación estrecha con el de autoorganización. Todo sistema complejo fluctúa, hasta quedar estabilizado temporalmente a partir del funcionamiento de sus partes, nexos y propiedades emergentes; esto se genera con la aparición de una serie de retroalimentaciones provocadas por los desafíos del entorno; externalidades sobre las que un sistema complejo tiene una incidencia relativa, pero que pretenden modificar su estructura (Gar- 
cía 2000). Los sistemas complejos no son lineales, unidimensionales y predictivos; son adaptativos, su capacidad de autoorganización les permite reaccionar a estímulos externos. ${ }^{2}$ Por tanto, el territorio, como uno de ellos, representa la capacidad fluctuante de autoorganización de los grupos sociales que, mediante formas contenido y acciones culturales, le dan significado a su ejercicio del ser-estar (Escobar 2005).

Al ser una propiedad emergente, la territorialización opera de manera dinámica, su adaptación a las circunstancias nuevas es permanente. La crisis se desencadena cuando quedan alteradas las interacciones de un territorio, debido al diálogo del paradigma o por la incidencia de algún evento externo significativo, lo que abre un tiempo incierto en el que las tendencias originarias y las recuperadoras de una estabilidad nueva se debaten incansablemente hasta lograr que emerja otra apropiación. Harvey (2000) describe a estos mecanismos como los que orientan la construcción social del territorio, y como los de estabilización que la regulan. En sentido temporal, la territorialización también es la proyección de las expectativas y aspiraciones sociales en un territorio, entendida como el ejercicio del derecho a seguir siendo-estando (Escobar 2005). Fernandes (2005) reconoce a los sistemas sociales y los socioterritoriales, como complejos, ya que conforman un mismo sujeto colectivo que se organiza para desarrollar una acción determinada en defensa de sus intereses, a través de enfrentamientos y acuerdos, cuyo objetivo es la trasformación de la realidad, y concluye que no son dos, sino que existen los sistemas sociales desde una perspectiva sociológica y los socioterritoriales, desde una geográfica.

Segunda tesis: la globalización demanda la reterritorialización de regiones específicas de acuerdo con los nuevos requerimientos del capital

En años recientes se han discutido ampliamente diversas expresiones sobre la globalidad, como la globalización, considerada como la 
influencia creciente de los procesos económicos, sociales y culturales mundiales sobre los nacionales y regionales (Comisión Económica para América Latina y el Caribe 2002, citado en Delgadillo 2006). Cuando se trata de abordar los fenómenos vinculados a los procesos de la globalización, el denominador común es la atención al territorio. Este retorno del territorio, como lo denominó Santos (2000), constituye un reflejo de la reestructuración mundial de las últimas décadas, después de la caída del bloque socialista. La relación globalización-territorio permite reconocer su connotación ideológica, ligada a las trasformaciones significativas (formas contenido y acciones) desarrolladas en la etapa actual del capitalismo.

De acuerdo con la concepción de Bauman (2003), la globalización ha vuelto líquido al territorio, puesto que el capitalismo concluyó una etapa de incrustación de los individuos en estructuras sólidas, como la producción industrial o las instituciones estatales. Desde esta perspectiva, la territorialización ha pasado a ser una especie de lastre, debido a sus efectos adversos sobre un sistema que basa su crecimiento en una movilidad frenética del capital. Para Rogerio Haesbaert (2004), este enfoque encierra las referencias a la desterritorialización, las cuales aluden al supuesto desarraigo del territorio como resultado de los cambios operados a través de la globalización. Aquí los dos fenómenos más importantes son: a) la reformulación del papel del Estado, que puso en crisis una serie de nociones asociadas a la soberanía, como referente territorial, y b) la "disminución" tecnológica de las distancias y el tiempo, que si bien no abatió los costes inherentes, sí facilitó una articulación nueva de lo local y lo global.

La globalización se manifiesta como un eje atravesado por fuerzas diversas articuladas en escalas anidadas (local, regional, global), lo cual sugiere la imposibilidad de dar cuenta de las identidades construidas, pues los límites no pueden definirse con claridad (Gómez y Hadad 2007). Según Raffestin (1993, citado en Schneider y Peyré 2006), esta comprensión permite pensar en un proceso denominado T-D-R (territorialización-desterritorialización-reterritorialización), asociado con el grado de acceso a la información, de símbolos o de significados múltiples, que pueden favorecer la construcción de territorios nuevos (territorialización), su deconstrucción (desterritorialización) o su reconstrucción (reterritorialización). Sin em- 
bargo, Haesbaert (2004) en varios trabajos polemiza contra el mito que representa la noción de desterritorialización, sobre todo porque resulta poco apropiado hablar de ésta sin referirse al mismo tiempo a una reterritorialización que le es inherente, y reconoce que el capitalismo contemporáneo no implica necesariamente un desarraigo de las identidades respecto del territorio.

Es paradójico que el proceso de planetarización, que duró casi cinco siglos, contenga una revitalización de la regionalización, mucho más antigua, y que fue la base inicial de la sobrevivencia y desarrollo de la especie humana (Serrano 1999). Se puede referir a dicha paradoja desde dos enfoques: a) el social, que alude al hecho de que una cultura globalizada debe cohabitar con otras diferenciadas, "la mundialización abriga en su seno su propia diferenciación inherente" (Gómez y Hadad 2007, 7) y b) el territorial, basado en las ideas de Milton Santos (2000), quien postula la presencia de los espacios de la globalización:

Hoy en día el lugar es un punto individualizado del acontecer global, $[\ldots]$ Cada lugar tiene una combinación horizontal de vectores verticales que son hijos de la globalización, pero que crean localmente una combinación específica y que definen una forma de vida específica. Ésta se realiza a través del lugar, que no es únicamente un receptáculo para recibir esa forma de vida, sino que es también un factor, un actor. El lugar no es sólo actuado por la globalización sino también y sobre todo, un actor de la globalización (Bosque y García 2003, 16).

Desde lo territorial, la globalización se presenta como un proceso que no abarca países completos sino espacios subnacionales, regiones, ciudades y áreas suburbanas (Harvey 2007b). "La globalización genera un nuevo tipo de relaciones entre las regiones, los gobiernos nacionales y los actores locales" (Delgadillo 2006, 1), ello brinda oportunidades y plantea riesgos originados en fuentes nuevas de inestabilidad, sobre todo en los ámbitos comercial y financiero, riesgos de exclusión para las regiones que no están preparadas para las fuertes demandas de competitividad neoliberal y riesgos de frag- 
mentación social y territorial dentro de países como México (Zurn 1998, citado en Beck 1998; Delgadillo 2006). Las fragmentaciones resultantes de la globalización hacen posible que procesos territoriales distintos puedan coincidir en una misma fase histórica, que obedecen a lógicas diferentes, y den como resultado una estructuración territorial más compleja y diversificada (Delgadillo et al. 2001).

Según Sloterdijk (2007), la historia de la globalización es la de una conquista doble: la de la tierra, por vía marítima, y la de la subjetividad, por la religiosa. Éstas dieron paso a una tercera, la del territorio interior, en ella los lugares se entrelazan a la vez que confunden su propia identidad, mientras se desplazan perdiendo su lugar original. Lo que se elogia o critica como globalización es la fase final de un proceso en el que el sistema mundial capitalista se ha desarrollado a plenitud condicionando todas las circunstancias de la vida, y privilegiado a ciertos países y sectores de la población. Sobre esta base, territorialmente hablando, la globalización significa la construcción de espacios exclusivos, ${ }^{3}$ es decir, los lugares beneficiarios resultantes de una reterritorialización acorde con los requerimientos del capitalismo en su fase neoliberal. En estas zonas, el Estado, el capital y la globalización están presentes ejerciendo una hegemonía con referentes, normas, instituciones y mercados; al mismo tiempo, existen lugares donde están presentes sólo en forma parcial y casi no son evidentes. Milton Santos describe esta condición como "una oposición entre espacios adaptados a las exigencias de las acciones económicas, políticas y culturales características de la globalización y otras áreas que no están dotadas de estas virtualidades" (Bosque y García 2003, 15). Las nuevas zonas de reterritorialización funcionan como los nodos o enclaves vinculados a la globalización, desde allí salen los productos a los mercados internacionales, pero también son el destino de las inversiones en capital y la trasferencia de tecnologías. Las ganancias que generan estos enclaves van de regreso hacia los territorios de sus propietarios y prestamistas estableciendo flujos de entrada y salida (Centro Latinoamericano de Estudios Superiores, ClaEs 2006). 
Tercera tesis: el lugar no es sólo actuado por la globalización sino que también y, sobre todo, es un actor en la globalidad

La habilidad de la globalización para resolver los problemas actuales cada vez es más cuestionada, se puede argumentar que no existen soluciones globales a muchos de los problemas de hoy (Santos 2000). "La inclusión selectiva y la hiper-exclusión de aumentada pobreza para los más y de inusitada riqueza para los menos" (Escobar 2005, 23) muestran una crisis de los modelos de pensamiento y las soluciones modernas que, al menos bajo la globalización, sólo agudizan los problemas.

En tanto que es un fenómeno global, el desarrollo geográfico desigual surge como un concepto que imbrica lo territorial y lo económico, y eso facilita la posibilidad de explicar los mecanismos por los que el capitalismo se reproduce a sí mismo desde un enfoque materialista-geográfico-dialéctico, no sólo desde uno histórico. ${ }^{4}$ Ello permite reconocer la forma en que se construyen las estructuras de poder territorial y las relaciones de éstas con el funcionamiento de las grandes trasnacionales o los flujos monetarios. La propuesta de Harvey (2007b) descarta la idea de utilizar la estructura de los Estados-nación como marco para comprender el desarrollo geográfico desigual, su base se refiere a analizar la competencia que se establece entre regiones, metrópolis y enclaves. La reterritorialización de los últimos años responde a la cuestión de cuáles son las escalas y los territorios más relevantes para el capital. La referencia a las escalas involucra la posibilidad de realizar recortes de la realidad, que son efecto de la aplicación de un esquema heurístico, o tratan de dar cuenta de su construcción social. Las escalas son el resultado de un proceso que involucra relaciones sociales de poder (económico, polí-

4 Para Harvey (2007b, 23): “La actividad capitalista está siempre fundada en algún lugar. Diversos procesos materiales (físicos, ecológicos, como también sociales) deben ser apropiados, usados, para los propósitos y caminos y sendas de la acumulación del capital. Recíprocamente, la acumulación del capital tiene que adaptarse y en algunos momentos transformarse por las condiciones materiales que encuentra. La teoría tiene que tener en cuenta dos problemas: primero, las reglas de la circulación y la acumulación del capital deben ser especificadas, y segundo, se debe establecer una metodología por la cual se pueda entender cómo estas reglas se vuelven tangiblemente expresadas y activamente reformadas a través de los procesos socio-ecológicos". 
tico, simbólico) y, por ende, de conflictos que se dirimen y expresan como niveles diferenciados de territorialización-reterritorialización. Lo que se deduce de ello es que la hegemonía no es un efecto de la escala, por el contrario, el proceso de construcción de una jerarquía deviene en un nivel territorial (Ortiz 2005).

Los conceptos lugar y local no son sinónimos (Swyngedouw 1998, citado en Escobar 2001). Lo local y lo global son escalas, procesos, pero no lugares. El lugar se refiere a la experiencia territorializada de y desde una locación particular, ligada a prácticas cotidianas realizadas por grupos de personas que, aunque heterogéneas y diversas, comparten lo que Virilio llama el hic et nunc (el aquí y el ahora) de la práctica social (1999, citado en Escobar 2005). La globalización, sugiere De Sousa, siempre es la globalidad exitosa de un lugar y, a partir de ello, propone una definición notable, es "el proceso por el cual una condición o entidad local dada, logra extender su alcance por todo el globo y, al hacerlo, desarrolla la capacidad de designar como local a alguna entidad o condición social rival" (2001, 4). Harvey (2005) explora esta dialéctica en la teoría de la acumulación por despojo, ${ }^{5}$ referida a los cambios ocurridos en la generación de rentas monopólicas en el capitalismo neoliberal. En el contexto de la globalización, éstas deben buscarse en las particularidades diferenciales entre territorios, en las marcas de distinción vinculadas a cada lugar; dichas rentas son esenciales para la reproducción del capital, pero se encuentran con la imposibilidad de convertir en mercancía a algunas reacciones incontrolables, que se oponen a la homogenización globalizadora. La buena noticia es que, para generar rentas monopólicas el capital debe tolerar la heterogeneidad y los particularismos territoriales y culturales (Harvey 2000). Es en esa contradicción donde el gran geógrafo británico identifica los espacios de esperanza, lugares en

5 Esta formulación teórico-metodológica supera las ideas de Lefebvre al respecto de que el capitalismo sobrevive a través de la producción del espacio. Harvey argumenta que la sobreacumulación en un sistema territorial supone un excedente de trabajo y de capital, los cuales pueden ser absorbidos por: “(a) el desplazamiento temporal a través de las inversiones de capital en proyectos de largo plazo o gastos sociales, los cuales difieren hacia el futuro la circulación de los excedentes actuales; (b) desplazamientos espaciales a través de la apertura de nuevos mercados, nuevas capacidades productivas y nuevas posibilidades de recursos y de trabajo en otros lugares; o (c) alguna combinación de (a) y (b)” (2005, 101). 
los que emerge una globalidad alternativa o no globalizada. ${ }^{6}$ En ellos surgen acciones y formas-contenido nuevas generadoras de rentas redistributivas, en las que todos los segmentos de la población se benefician del capital. Entre los casos destacables, Harvey ubica el Foro Social Mundial, los municipios autónomos zapatistas, las ciudades de Porto Alegre, Hong Kong y Singapur, en donde hay una apropiación pública de las rentas del suelo. Estos territorios constituyen un triunfo para los movimientos sociales, al ser la razón de su existencia (Raffestin 1993); la "otra" reterritorialización que representan, es esencial para entender el contexto global actual. Gonçalves (2001) reconoce la existencia de una tensión de territorialidades, porque no hay una dinámica global unidireccional; en la dialógica geográfica del capitalismo actual, los lugares están marcados por la diversidad. Fernandes (2005) profundiza en esta interpretación, y al analizar los movimientos sociales con base en sus alcances y estructuración territorial, establece la existencia de los socioterritoriales aislados y territorializados.

David Harvey (2000) propone Edilia, un ejercicio de imaginación en donde se esboza una posible sociedad poscapitalista, una suerte de "socialismo de mercado"7 en donde la distribución del valor no se da necesariamente a partir de la propiedad de los medios de producción, ni se cuestiona la vinculación que el lugar tiene con otros y con la globalidad; por el contrario, es el lugar donde se definen las formas de redistribución equitativa de la renta derivada de la interacción con el mercado, esta construcción socioterritorial hace énfasis en la mediación de los asuntos de interés público basada en lo que Reclus (1905) y Kropotkin (1902) identificaron como cooperación y apoyo mutuo. Los espacios de esperanza devienen de la emergencia de formas de pensar y actuar diferente a las de la globalización, propias de cada lugar y alternativas a las hegemónicas; el sociólogo

6 Las nociones de espacios exclusivos y espacios de esperanza son insuficientes para explicar los tipos de territorios existentes, en principio, un tercer tipo, que podría denominarse espacios de desesperanza, implicaría a los que no son lugares de la globalización, pero que tampoco son alternativos a ésta.

7 Harvey (2007a) afirma que identificar el capitalismo con el mercado es un error, tanto como confundir dinero con capital. 
portugués Boaventura De Sousa (2009) las denomina epistemologías del sur. ${ }^{8}$

Hay lugares exclusivos de la globalización, con sus enclaves, tramas y redes que apuestan a controlar el futuro desde la seguridad de la ciencia, la modernidad y el desarrollo; y también existen los emergentes, los espacios de esperanza, cada uno con su epistemología, su identidad y su imaginación, que apuestan por un futuro en muchos aspectos incierto, alternativo y posdesarrollista. Los primeros están estructurados a partir de sistemas globales (capitalismo, colonialismo, patriarcado, extractivismo, adultocentrismo), que mantienen su poder hegemónico explotando, discriminando, oprimiendo, deteriorando y excluyendo a "los otros". En los segundos, el anclaje socioterritorial busca liberarse de manera parcial, imprecisa y creativa del condicionamiento de los sistemas mundo, por ello buscan ser alternativos, diversificados, es decir, antisistémicos (Wallerstein 2006), híbridos e incluyentes, que transitan de la noción de "los otros" hacia la construcción del "nosotros". En ese trayecto, el lugar se resignifica como actor en la globalidad, a través de la emergencia de nociones diversas que devienen en un complejo proceso social superior, a las que Freire (1970) denominó acciones dialógicas; esta perspectiva multiplica los territorios hacia posibilidades y territorialidades infinitas, imaginables, pero impredecibles.

Cuarta tesis: la globalización impulsa la noción de desarrollo sustentable como sinónimo de progreso; los movimientos sociales emergentes proponen la sustentabilidad desde el ámbito local

El Informe Brundtland, presentado el 4 de agosto de 1987 con el nombre de Nuestro futuro común, proclamaba la necesidad de trabajar en la dirección de un desarrollo sustentable. Esta propuesta fue un intento para afrontar un doble desafío de la humanidad: la con-

8 "Las Epistemologías del Sur son el reclamo de nuevos procesos de producción, de valorización de conocimientos válidos, científicos y no científicos, y de nuevas relaciones entre diferentes tipos de conocimiento, a partir de las prácticas de las clases y grupos sociales que han sufrido, de manera sistemática, destrucción, opresión y discriminación causadas por el capitalismo, el colonialismo y todas las naturalizaciones de la desigualdad en las que se han desdoblado” (De Sousa 2009, 16). 
dición de pobreza en que vive la mayoría de la población y los retos planteados por los problemas ambientales; este proceso debe de ser capaz de generar un desarrollo equilibrado en términos ecológicos, sociales y económicos, se define como "el desarrollo que satisface las necesidades de la generación presente sin comprometer la capacidad de las generaciones futuras para satisfacer sus necesidades" (United Nations 1987, 37).

En este marco se asume que "el carácter sostenible es, en un sentido muy amplio, una cuestión de asegurar una equidad de la distribución, de compartir las oportunidades de desarrollo entre las generaciones actuales y las futuras" (Programa de las Naciones Unidas para el Desarrollo, PNUD 1995, 15); premisa que estableció un problema ético del concepto con el pasado, pues las generaciones anteriores acumularon deudas económicas, ecológicas y sociales cuya factura se cobra hoy. Al respecto, el PNUD planteó un dato contundente, "la repetición de las pautas de consumo del Norte en el Sur requeriría 10 veces las existencias de minerales; es claro que el estilo de vida de los países ricos tendrá que cambiar" $(1995,20)$. Existe otro problema con respecto al futuro, ya que no hay manera de imaginar si son aceptables o no los estilos de vida actuales, y si hay alguna razón para trasmitirlos a las generaciones siguientes. El Informe Bruntdland pretendió establecer la noción de desarrollo sustentable como un medio para preservar al desarrollo9 "de su contradicción con el ambiente y los recursos naturales" (Sachs 1996, citado en Demo et al. 1999, 14), con el propósito de evitar que el ambiente se convierta en una limitante para el desarrollo económico, para ello fue necesario postular principios, protocolos y fórmulas para medir al desarrollo sustentable. La propuesta de Giglo (1997) se orienta, por primera vez, en esa dirección, al sugerir la posibilidad de estabilizar un sistema socioambiental con base en estrategias que tiendan a neutralizar o minimizar los disturbios ocasionados de manera antrópica. En apoyo a ello, Lyon (1996) propuso lineamientos e indicadores generales del desarrollo sustentable, el Marco de Evaluación de

9 “Simbólicamente, el desarrollo está ligado a una promesa de bienestar, de felicidad, de calidad de vida $[\ldots]$ ata irremediablemente a un imaginario determinado, occidental, capitalista y colonial; ya que pretende que los excluidos sigan un camino pre-trazado por el Norte global para lograr su inclusión en el modo de vida hegemónico” (Lang y Mokrani 2012, 13). 
Sistemas de Manejo de Recursos Naturales, incorpora indicadores de sustentabilidad (Masera et al. 1999), el Banco Mundial (Demo et al. 1999) o el PNUD, que a partir de 1990 publica informes anuales de desarrollo humano; en la actualidad existen infinidad de propuestas orientadas a conocer lo cerca o lejos que se está de "ser sustentable".

A partir de la Cumbre de Río, en 1992 y de su secuela, en Johannesburgo, se formalizó lo que Gonçalves (2001) denomina geografía política del desarrollo sustentable, que devuelve la cuestión ambiental a la exclusión mutua sociedad-naturaleza; al insertar la noción de desarrollo con un enfoque teleológico otorga "propósitos" propios a los hechos y fenómenos naturales, y así deslinda la responsabilidad social con la naturaleza. En este marco, el desarrollo sustentable opera como un mecanismo de la globalización anclado en una nueva geografía política que deja a un lado la soberanía de los Estados nacionales, para sustituirla por las orientaciones de organismos internacionales (Delgadillo 2006).

Cuando Holling $(2001,403)$ planteó que "la sustentabilidad requiere tanto de cambio como de persistencia”, emergió un paradigma nuevo que cuestiona la esencia del desarrollo sustentable como el epítome del equilibrio. Al respecto, Jules Pretty (1995) propone cinco elementos que permiten caracterizar la construcción de sustentabilidad desde una perspectiva social: a) no se puede definir de manera precisa; b) los problemas siempre están abiertos a su interpretación; c) la solución de un problema genera otros; d) la clave para la atención de los problemas deviene de la capacidad de los actores para aprender en condiciones de cambio permanente y e) los sistemas de interacción y aprendizaje son necesarios para hacer coincidir los intereses diversos. Por tanto, la sustentabilidad es un debate siempre abierto, un proceso flexible derivado de una responsabilidad colectiva, la cual logra criterios consensuados y construye alternativas que se desprenden de un requerimiento permanente de conocimientos nuevos (Roling y Wagemakers 2000).

$\mathrm{Al}$ igual que la territorialización, la sustentabilidad es una propiedad emergente de un sistema complejo (Woodhill y Roling 2000). Cuando se logran acuerdos sobre lo que la gente quiere aprovechar de manera sustentable en un lugar específico, la mitad del trabajo está hecho (Roling y Wagemakers 2000), la otra es llevarlos a cabo como 
parte de un programa social. Desde esta perspectiva, los aciertos y desaciertos del Informe Bruntdland pueden resignificarse dejando atrás la noción del desarrollo sustentable como un postulado euclidiano, y sustituirla por construcción de las sustentabilidades, que representa mejor los cambios profundos que ocurren a través de la implementación de acciones dialógicas en el ambiente.

El enfoque territorial se presenta como una categoría provista por la geografía para enriquecer las discusiones acerca de la sustentabilidad. En la escala local, por ejemplo, se puede reconocer con claridad cómo los procesos sociales construyen territorios y sustentabilidades; es donde se estructuran los sistemas sociales, las políticas gubernamentales, los organismos internacionales e incluso el mercado, los cuales, mediante mecanismos de consenso responden a problemáticas concretas, que a su vez tienen efectos verticales y horizontales en el ámbito doméstico, regional y global.

La construcción de sustentabilidades está asociada con una toma de decisiones continua y cambiante orientada a mejorar el "desempeño" territorial de regiones, lugares y redes en escalas múltiples. Es un proceso complejo, que asume que la base de la trasformación socioterritorial deriva del trabajo crítico y organizado, en el cual se garantiza la reproducción material y cultural con una visión de largo plazo, en un marco de equidad que deviene del ejercicio participativo, propio de cada lugar (Arreola y Saldívar 2006; Bassols 1986). La construcción de las sustentabilidades también sugiere la necesidad de desplazarse de la sociología de los conocimientos subalternos a la política de surgimiento de los movimientos sociales (Leff 2003a), que tienden a mostrar propiedades emergentes y un comportamiento adaptativo complejo, que los del pasado nunca fueron capaces de manifestar, debido a su centralización y jerarquía. Esta lógica es reforzada por las dinámicas de autoorganización basadas en las nuevas tecnologías de información y comunicación (Castells 2006).

Estas construcciones de las sustentabilidades, situadas en un lado contrapuesto a los espacios exclusivos de la globalización, articulan prácticas de la diferencia social, económica y ecológica que son útiles para pensar sobre mundos locales y regionales alternativos (Leff 2003a). Los movimientos socioterritoriales como los Sin Tierra, que crean islotes autogestionados; los indígenas ecuatorianos, que re- 
construyen sus territorios étnicos ancestrales y los indígenas chiapanecos, que conforman municipios autónomos zapatistas (Fernandes 2000; Ramón 1993; García de León 2002, citados en Zibechi 2003) son algunos mundos alternativos, caracterizados por su complejidad, interiorización e incertidumbre. La representación social que significan está basada en contratos individuales y comunitarios ancestrales o innovadores: una nueva acción dialógica local y global, que se construye como alternativa al desarrollo. ${ }^{10}$

Quinta tesis: los movimientos socioterritoriales híbridos construyen un proceso de adaptación complejo, que reterritorializa redes de lugares hacia la sustentabilidad

El territorio se recrea dinámica y permanentemente en convivencias trascendentes y efímeras, cuyas formas, contenidos, reglas, funcionamientos y dirección se sostienen mediante procesos sociohistóricos, en donde las posibilidades de permanencia dependen de las potencialidades para sostener procesos locales y globales, según su propia funcionalidad y dialéctica (Santos 2000).

En cada momento, el proceso socioterritorial involucra una redistribución de sus factores, y en cada lugar el tiempo actual se enfrenta con el tiempo pasado, donde el trabajo ya hecho se impone sobre el trabajo por hacer, es decir, que su distribución territorial actual descansa sobre sus divisiones anteriores.

La comprensión de los lugares en su situación actual y en su transformación depende de la consideración del eje de las sucesiones y del eje de las coexistencias, donde se reconoce que el territorio es el que reúne a todos, con sus múltiples posibilidades, que son posibilidades diferentes de uso del espacio relacionadas con posibilidades diferentes de uso del tiempo (Santos 2000, 134-135).

10 Esta afirmación se basa en la formulada por Gudynas $(2012,42)$ en el sentido de que "sea necesario distinguir entre los 'desarrollos alternativos' de las 'alternativas al desarrollo'. El primer caso sirve para las distintas opciones de rectificación, reparación o modificación del desarrollo contemporáneo, donde se aceptan sus bases conceptuales, tales como el crecimiento perpetuo o la apropiación de la Naturaleza, y la discusión se enfoca en la instrumentalización de ese proceso. En cambio, las 'alternativas al desarrollo' apuntan a generar otros marcos conceptuales a esa base ideológica. Es explorar otros ordenamientos sociales, económicos y políticos de lo que veníamos llamando desarrollo". 
En cada momento siempre hay un mosaico imbricado, una red sobrepuesta y múltiple de territorios cubriendo la superficie de la Tierra cuyo diseño lo proporciona el curso de la historia; de tal forma, la escala deja de ser una noción geométrica para ser condicionada por el tiempo.

Las redes serían incomprensibles si sólo se vislumbraran a partir de sus manifestaciones locales o regionales. Son el vehículo de un movimiento dialéctico que posiciona a los lugares en el mundo, y hace que enfrenten los desafíos globales. Hoy las redes sugieren dos tipos de construcciones territoriales: a) las extensiones formadas por lugares que se agregan sin discontinuidad, como en la definición tradicional de la región y b) los lugares que, separados unos de otros, aseguran el funcionamiento global de la sociedad y de la economía (Santos 2000). Para clarificar estos conceptos, Fernandes (2009) propone las siguientes denominaciones: a) un primer territorio, los espacios de gobernanza, que han sido predominantemente sólidos (municipios, barrios, localidades, ejidos); b) las propiedades sobre la tierra, que constituyen el segundo territorio, son los espacios vividos de representación (empresas, comunidades, grupos indígenas, asociaciones de vecinos), que pueden ser sólidos o líquidos y e) el tercer territorio, el espacio relacional, es decir, las redes líquidas (movimientos sociales, trasnacionales, redes basadas en tecnologías de información y comunicación).

Esta reticularización del territorio (Hiernaux 1999) es la red en donde todos los lugares tienen propósitos definidos a partir de los intereses individuales, y también de la sistemática compleja que opera a través de acciones dialógicas, que se alimentan mutuamente. Desde esta perspectiva, el tercer territorio constituye un proceso social superior, puesto que las acciones se entrelazan, suceden, coexisten y fluyen por medio de cadenas dialógicas. Ahora bien, dentro de la red, la jerarquía no está asegurada de modo alguno (Escobar 2005), ni es estable, está mediada por la entropía. La gente sigue viviendo en lugares, pero como en las sociedades la función y el poder se organizan en el espacio de los flujos, su lógica altera, de forma esencial, el significado y la dinámica de los lugares (Castells 2006). Por tanto, el tercer territorio es el relacional, reúne a todos los demás tipos, une las propiedades fijas y móviles, promueve la 
expansión y el reflujo condicionado por las relaciones sociales y los conflictos entre las clases, grupos, individuos, mercado y Estado. No está subordinado a las condiciones de gobierno ni de propiedad, que caracterizan a los otros dos territorios, se relaciona primordialmente con las formas-contenido y las acciones que se entrecruzan e intercambian, y constituyen la red más sublime del continuo de la vida cotidiana (Harvey 2007a). Esto significa una transición epistemológica desde la geografía política, de las líneas y polígonos, hacia la geografía política de las redes, a partir de la cual Hiernaux y Lindón (2012) proponen abordar lo imaginario, como una dimensión de las geografías de la vida cotidiana.

Uno de los mejores esfuerzos por explicar dicho continuo es el de Gunderson y Holling (2002), quienes desde el enfoque de los sistemas complejos, y basados en décadas de estudio sobre los ecosistemas y sus efectos en la gestión ambiental y social, formularon un modelo que da una idea del proceso de cambio reconocible a lo largo de todas las escalas, desde los individuos a los biomas, las familias a las culturas y los días a los siglos, que es conocido como ciclo de adaptación, ${ }^{11}$ la metáfora original (Holling et al. 2002) lo ilustra con el símbolo matemático de infinito $(\infty)$, que se extiende en dos dimensiones: el incremento de la conectividad y del capital almacenado. La conectividad se refiere a la cantidad de asociaciones entre elementos y procesos del sistema complejo, representa la unión producto de la tensión entre las fuerzas de estabilización y desestabilización, refleja la rigidez o flexibilidad de las variables y de los procesos activos. El capital almacenado o potencial se refiere a la suma de los recursos disponibles dentro del sistema, determina su productividad actual y la amplitud de sus opciones futuras (Winkle 2011, citado en Jiménez 2013). Las cuatro fases que lo integran constituyen los elementos de un ciclo de cambio adaptativo (véase figura 1): explotación $(\mathrm{r})$, conservación $(\mathrm{K})$, liberación $(\Omega)$ y reorganización $(\alpha)$. Desde un punto de vista social, dichas fases pueden definirse como: (r) emprendimiento (una organización política y social nueva); (K)

11 Hay sistemas complejos que tienen la capacidad para autoorganizarse y aprender, durante los procesos que los definen, se les denomina complex adaptative systems (sistemas adaptativos complejos), este es el caso de los socioterritoriales (Sáez et al. 2003, citado en Jiménez 2013). 
consolidación organizativa (mantenimiento y la proliferación del sistema nuevo); $(\Omega)$ liberación creativa (revolución) y $(\alpha)$ reorganización (cambio de régimen). El modelo permite también equiparar las sucesiones desde la perspectiva territorial descrita en las hipótesis precedentes: apropiación territorial (r), territorialización $(\mathrm{K})$, fragmentación $(\Omega)$ y reterritorialización $(\alpha)$.

El modelo del ciclo de adaptación permite interpretar los procesos de un sistema socioterritorial complejo, que se suceden a través de adaptaciones internas y con el entorno; esto, por medio del reconocimiento de las acciones y cadenas dialógicas que construyen, según una lógica que va adquiriendo o perdiendo su carácter a lo largo del tiempo, como ocurre en los espacios exclusivos, en los emergentes y en los de esperanza. Para ello, además de las fases descritas, el modelo incluye también cuatro transiciones o flujos.

La transición ( $\mathrm{r}-\mathrm{K}$ ) es un periodo de expansión paulatina con un comportamiento relativamente predecible. Aquí la conectividad y

Figura 1

El ciclo de adaptación

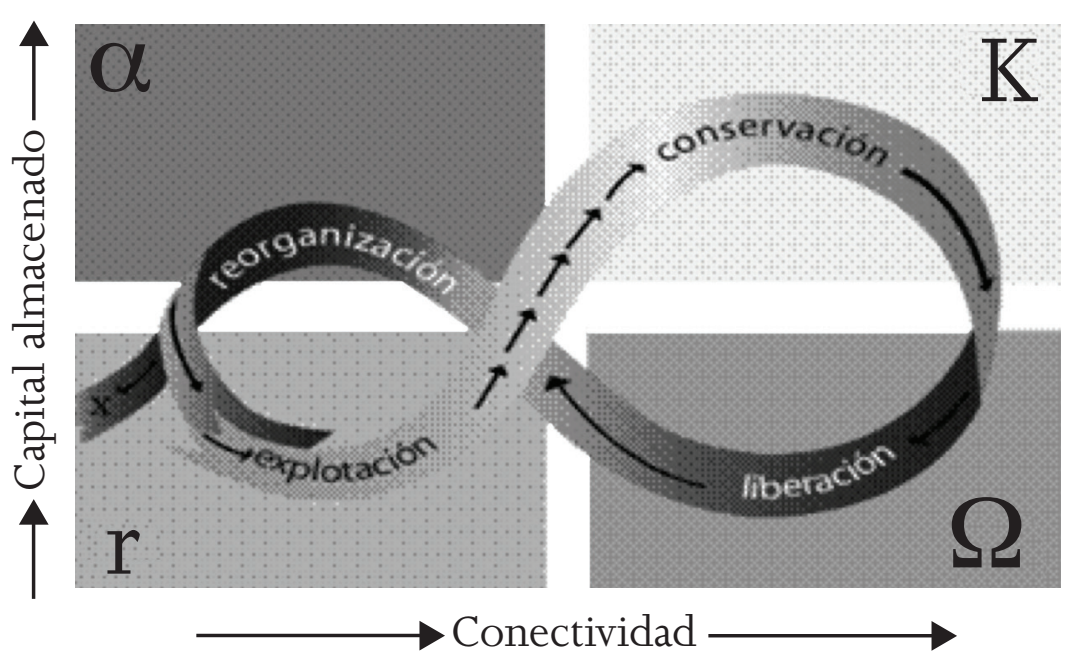

Fuente: Gunderson y Holling (2002). 
la estabilidad crecientes del sistema incrementan la confianza entre elementos y procesos, de modo que representa un potencial creativo (por ejemplo, mayor disponibilidad de nutrientes, de producción de biomasa, de innovación en las habilidades y en las relaciones humanas). Aquí se refuerza la especialización, lo que hace más eficiente el sistema (Jiménez 2013). Las propiedades emergentes establecidas en la fase (r) se trasforman cualitativamente en acciones dialógicas en la $(\mathrm{K})$, y ponen en marcha el mecanismo de apropiación-territorialización.

En la transición (K- $\Omega$ ) ocurre una deconstrucción progresiva, en ella la incertidumbre se incrementa, ya que la conectividad y estabilidad requieren dispositivos temporales para sostener la prevalencia de los elementos que ejercieron un dominio en la fase (K). La erosión, el cambio generacional, el crecimiento en el consumo energético, la sustitución de procesos productivos, el cambio de uso del suelo, por citar algunos casos, revelan tensiones entre las fuerzas que quieren mantener el status quo y las que lo deterioran o cuestionan. En esta transición, la capacidad de autoorganización del sistema disminuye su eficiencia debido a una reducción del potencial creativo; aparecen nociones dialógicas nuevas, que emergieron del interior del sistema o del entorno. Esto implica el paso de la territorialización a la fragmentación territorial.

La transición $(\Omega-\alpha)$ es caótica, porque la cohesión social y la conectividad tienden a menguar drásticamente, pues los dispositivos que estabilizaban al sistema dejan de funcionar. La trayectoria está significada por el cambio, la destrucción, la renovación y la innovación en un contexto de gran incertidumbre, en el que eventos aleatorios menores ofrecen la oportunidad de conformar una identidad nueva y definir otra trayectoria (Walker y Salt 2006, citados en Jiménez 2013). La vulnerabilidad interna crece a tal grado, que la influencia de las externalidades se incrementa como en ninguna otra transición. Los mecanismos que ponen en marcha la fragmentaciónreterritorialización abren el camino a las nociones y acciones emergentes con capacidad trasformadora, evidenciada como alternativa a una hegemonía decadente; tal es el caso de la producción y el mercado orgánicos, el comercio justo, los movimientos sociales que vigilan el ejercicio de los derechos humanos, laborales, de mujeres, niñas y 
niños, el rescate de espacios públicos y el uso de las redes sociales para la movilización democrática.

La transición $(\alpha-r)$ es un periodo de reconstrucción, en el que la conectividad y el capital almacenado del sistema tienden a ser escasos, lo que permite que la acción dialógica mejor posicionada dé paso a un ciclo cualitativamente superior o al reinicio del ciclo establecido. En ella se construyen los consensos que confirman una hegemonía nueva, que deja su carácter emergente. En este trayecto ocurren cambios legales, en el paisaje y en los ecosistemas, de adscripción política, de tecnología y orientación en el mercado. A la reterritorialización de la fase $(\alpha)$ le sigue una apropiación nueva del territorio en la (r).

El ciclo de adaptación permite explicar la complejidad de los procesos socioterritoriales, ya que tanto fases como transiciones trascurren en el tiempo en las escalas geológicas, históricas y humanas. Cada ciclo puede tener efectos en otros concurrentes, espacial y temporalmente (Weeks et al. 2004), lo cual permite el surgimiento de transiciones que no están sujetas al ciclo $(\infty) .{ }^{12}$

Desde el punto de vista territorial, cada ciclo individual se encuentra anidado con otros en diversos niveles (Holling 2003, citado en Jiménez 2013); ningún ciclo de adaptación se puede considerar en un nivel único, ya que la importancia de las relaciones interniveles es fundamental en su dinámica (Resilience Alliance 2013). A esta articulación de escalas temporales y niveles territoriales interconectados Gunderson y Holling (2002) la denominan panarquía. ${ }^{13}$

El funcionamiento heterárquico de los ciclos de adaptación anidados influye poderosamente en la sustentabilidad de un sistema socioterritorial (Holling 2001). Esto se explica debido a que la dinámica de una fase en un nivel puede propagarse a una distinta en

12 Hay flujos $(\mathrm{r}-\Omega)$ que suponen nociones dialógicas no consolidadas, y son desechadas, (K-r) que sugiere un retroceso desde la fase de conservación a la de emprendimiento, y $(\alpha-\Omega)$ que reconoce un flujo regresivo desde la fase de reorganización, en donde una acción emergente no logra el consenso suficiente y no llega a ser hegemónica.

13 Puydt (1860) fue el primero que utilizó el concepto de panarquía desde el punto de vista político y económico, al referirse a un Estado en el que los individuos y las instituciones ejercen libremente sus derechos y, por tanto, se puede descartar la necesidad de un gobierno. Por su vínculo con las posiciones políticas del anarquismo, se prefiere usar la palabra panarquismo para referirse a esta definición. 
otro, y amplificarla o atenuarla, y desafiar al sistema socioterritorial en una vía dirigida o no al cambio. El ciclo de adaptación es un recurso heurístico que permite reconocer a los actores sociales, y a las nociones emergentes que alientan la construcción de la sustentabilidad en cada lugar. En este sentido, Cortés (2012) propone: a) identificar y destruir las restricciones perniciosas y las inhibiciones del cambio; b) proteger y preservar la acumulación de experiencias en las que se basa cada cambio; c) estimular la innovación y comunicar resultados con una variedad de experimentos, para probar direcciones que reduzcan los costos organizacionales del cambio; d) animar la aparición de entidades para renovar y sostener la acción colectiva, el desenvolvimiento económico y el pacto social, a través del cambio, y e) promover programas que fomenten el conocimiento de las experiencias trasformadoras entre los ciudadanos, las organizaciones sociales, las empresas y los órdenes de gobierno, para que se comprometan con el cambio. Si la sustentabilidad como alternativa significa alguna cosa, tiene que ver con las cadenas dialógicas y las trasformaciones que pueden ocurrir durante el proceso de adaptación de un sistema socioterritorial complejo en un lugar.

\section{Conclusiones}

En el campo de las alternativas al desarrollo se ha experimentado mucho en diversos lugares, en un intento por combinar conocimiento y poder, para incorporar activamente a los movimientos socioterritoriales.

¿Cómo el conocimiento local será traducido en poder y este saberpoder en proyectos y programas concretos?, un primer paso es hacer visibles las diferentes lógicas locales de producción de culturas e identidades, de prácticas ecológicas y económicas que incesantemente están emergiendo en lugares del mundo entero. ¿Hasta dónde logran plantear un importante, y quizás original, reto al capitalismo y la modernidad? Más aún, ¿cuáles serían las condiciones que permitirían a las prácticas basadas en el lugar crear estructuras alternativas que les den una oportunidad para sobrevivir, crecer por sí mismas y florecer? (Escobar 2005, 179). 
Existen referentes que permiten reconocer la existencia de movimientos socioterritoriales, que viran en una dirección alternativa orientada a la sustentabilidad, basados en instituciones comunitarias de larga duración (Ostrom 2000; Merino 1999), que se asocian a regiones donde existen derechos de propiedad establecidos. Estos lugares, en donde se construyen espacios de esperanza ya son una realidad: las organizaciones de producción orgánica, las comunidades que manejan sustentablemente sus bosques y selvas, las redes de reservas ecológicas comunitarias de centros ecoturísticos, los municipios autónomos zapatistas. La resignificación de estos lugares se enfrenta, en el campo de las luchas políticas, con múltiples hegemonías de los sistemas-mundo, la emergencia de cadenas dialógicas a partir de la hibridez entre lugares podría, en todo caso, facilitar la construcción de una globalidad de esperanza, la reterritorialización que implica está inscrita en la ecología política que, como todas, no sólo es una estrategia práctica, sino también una lucha que se da en la producción y apropiación de las ideas (Leff et al. 2002).

Es en esta epistemología que los conceptos de territorio-región funcionan como lugares-soporte, para la reconstrucción de identidades enraizadas en prácticas culturales y racionalidades productivas sustentables (Leff 2003b). En este caso, el diálogo del paradigma es uno de saberes ${ }^{14}$ sobre el territorio, fundamental para la creación y recreación de las prácticas ecológicas, económicas y culturales de los lugares. En esta misma perspectiva, la geografía cultural da valor a la diversidad manifiesta en todos los paisajes de la superficie terrestre. Fernández (2006) y el enfoque intercultural de Argueta (2011) advierten sobre la importancia de que los sistemas de los pueblos originarios se sigan construyendo desde sus propias historias y epistemologías, para asegurar, de forma efectiva, diálogos de saberes donde se pueda garantizar la construcción de una sociedad plurinacional, pluriétnica y multilingüe, sustentada en el respeto a la diversidad. En esta articulación entre identidad y reterritorialización

14 "La aproximación que se hace al diálogo de saberes, en este texto, apunta a entenderlo como un tipo de hermenéutica colectiva, donde la interacción caracterizada por lo dialógico recontextualiza y resignifica los dispositivos pedagógicos e investigativos que facilitan la reflexividad y la construcción de sentidos de los procesos, acciones, saberes, historias y territorialidades" (Ghiso 2000, 1). 
subyace la ecología política de los movimientos socioterritoriales emergentes orientados hacia la sustentabilidad, que se presentan con rostros diversos.

Con el modelo del ciclo de adaptación es posible reconocer que la construcción de los movimientos socioterritoriales es continua, que los lugares siempre se definen y recrean, y que hay propuestas que emergen en busca de ser, estar y seguir siendo-estando. En última instancia, la nueva geografía y ecología política pueden contribuir a reinstaurar la crítica a las hegemonías actuales, como una pregunta de imaginación utópica tal y como en su momento lo hicieron Reclus (1905) y Harvey (2000).

¿Puede el mundo ser reconcebido y reconstruido desde la perspectiva de una multiplicidad de prácticas de cultura, ambiente y economía basadas en el lugar? ¿Qué formas de lo global pueden ser imaginadas desde múltiples perspectivas basadas en el lugar? ¿Qué contra-estructuras pueden ser puestas en práctica para volverlas viables y productivas? ¿Qué nociones de política y democracia son necesarias para hacer efectivo al lugar en todas sus multiplicidades y contradicciones? ¿Qué rol deben jugar los diferentes actores sociales, incluyendo a las viejas y nuevas tecnologías, para permitir crear redes en las cuales las diversas formas del lugar puedan encontrarse con las múltiples manifestaciones de lo global? (Escobar 2005, 193).

El campo de los movimientos socioterritoriales, en particular de algunos étnicos y ambientales, ofrece un terreno fértil para pensar en estas preguntas y en las políticas del lugar en general. Por ejemplo, la noción del territorio que están elaborando los activistas de diversos movimientos alternativos establece relaciones nuevas entre el lugar, los géneros, las generaciones, la cultura y el ambiente. El punto consiste en distinguir las formas de globalización que pueden convertirse en fuerzas políticas efectivas hacia lo local, para la defensa del lugar y de las identidades, así como las formas de localización que, desde el lugar, pueden llegar a utilizar su propia ventaja en lo global. Como Virilio (1999) lo expresó, "amo lo local cuando le permite a 
uno ver lo global y amo lo local cuando uno lo puede ver desde lo global" (citado en Escobar 2005, 177).

Recibido en febrero de 2014 Aceptado en junio de 2015

\section{Bibliografía}

Argueta, Arturo. 2011. El diálogo de saberes, una utopía realista. Integra Educativa v (3): 15-29.

Arreola, Arturo y Antonio Saldívar. 2006. Entre lo territorial y lo sectorial: la experiencia de las microregiones en la selva Lacandona, Chiapas. Revista de Geografía Agrícola (37): 57-78.

Bassols, Ángel. 1986. Lucha por el espacio social. Regiones del norte y noreste de México. México: Universidad Nacional Autónoma de México (UNAM).

Bauman, Zygmunt. 2003. Modernidad líquida. México: Fondo de Cultura Económica (FCE).

Beck, Ulrich. 1998. ¿Qué es la globalización? Falacias del globalismo respuestas a la globalización. Barcelona: Paidós Ibérica.

Bosque, Joaquín y Aurora García. 2003. Milton de Almeida Santos (1926-2001). Anales de Geografía de la Universidad Complutense (23): 9-19.

Castells, Manuel. 2006. La sociedad red. Madrid: Alianza.

CLAES. 2006. Integración regional, globalización y opciones alternativas. https://www.google.com.mx/\#q=CLAES.+2006.+Integrac $\mathrm{i} \% \mathrm{C} 3 \% \mathrm{~B} 3 \mathrm{n}+$ regional $\% 2 \mathrm{C}+$ globalizaci $\% \mathrm{C} 3 \% \mathrm{~B} 3 \mathrm{n}+\mathrm{y}+$ opciones+ alternativas (29 de marzo de 2012).

Cortés, Raúl. 2012. Entendiendo la complejidad de la economía, la 
ecología y los sistemas sociales. Administración \& Desarrollo 40 (55): 117-120.

De Sousa, Boaventura. 2009. Una epistemología del Sur: la reinvención del conocimiento y la emancipación social. México: Siglo XXI, CLACSO.

De Sousa, Boaventura. 2001. Nuestra América. Reinventando un paradigma subalterno de reconocimiento y redistribución. Revista Chiapas (12): 179-201.

Del Carpio, Carlos. 2003. Apropiación social del territorio en la frontera Chimalapa. Tuxtla Gutiérrez: Universidad Autónoma de Chiapas.

Delgadillo, Javier. 2006. Reestructuración económica territorial en el marco de la globalización. Geoecon Revista Virtual de Geografía Económica (1): 13-26.

Delgadillo, Javier, Javier Torres y Felipe Gasca. 2001. El desarrollo regional de México en el vértice de dos milenios. México: Miguel Ángel Porrúa, UNAM.

Demo, Claudio, Guillermo Montoya, Luis García y Alejandro Morón.1999. El Banco Mundial y el desarrollo sustentable. Algunas reflexiones sobre su perspectiva. Problemas del Desarrollo 30 (118): 9-34.

Escobar, Arturo. 2005. Más allá del Tercer Mundo. Globalización y diferencia. Bogotá: Instituto Colombiano de Antropología e Historia.

Escobar, Arturo. 2001. Culture sits in places: reflections on globalism and subaltern strategies of localization. Political Geography (20): 139-174.

Fernandes, Bernardo. 2009. Sobre la tipología de los territorios. http://www.acciontierra.org/spip/spip.php?article420 (14 de octubre de 2013). 
Fernandes, Bernardo. 2005. Movimientos socioterritoriales y movimientos socioespaciales. Observatorio Social de América Latina (16): 273-284.

Fernández, Federico. 2006. Geografía cultural. En Tratado de geografía humana, compilado por Daniel Hiernaux y Alicia Lindón, 220252. México: Universidad Autónoma Metropolitana Iztapalapa (UAM-I).

Freire, Paulo. 1970. La pedagogía del oprimido. México: Siglo XXI.

García, Rolando. 2000. El conocimiento en construcción. De las formulaciones de Jean Piaget a la teoría de sistemas complejos. Barcelona: Gedisa.

García, Rolando. 1986. Conceptos básicos para el estudio de sistemas complejos. En Los problemas del conocimiento y la perspectiva ambiental del desarrollo, compilado por Enrique Leff, 45-71. México: Siglo XXI.

Ghiso, Alfredo. 2000. Potenciando la diversidad (diálogo de saberes, una práctica hermenéutica colectiva). http://bibliotecadigital. conevyt.org.mx/colecciones/documentos/potenciando_diversidad.pdf (17 de marzo de 2014).

Giglo, Nicolo. 1997. En torno a la sustentabilidad ambiental del desarrollo agrícola latinoamericano: factores y políticas. En El futuro ecológico de un continente. Una visión prospectiva de la América Latina, compilado por G.C. Gallopín. México: FCE.

Gómez, César y Gisela Hadad. 2007. Territorio e identidad. Reflexiones sobre la construcción de territorialidad en los movimientos sociales latinoamericanos. http://webiigg.sociales.uba.ar/iigg/ jovenes_investigadores/4jornadasjovenes/EJES/Eje\%206\%20Espacio\%20social\%20Tiempo\%20Territorio/Ponencias/HADAD_ Gisela.pdf (4 de noviembre de 2013).

Gonçalves, Carlos. 2001. Geografías. Movimientos sociales, nuevas territorialidades y sustentabilidad. México: Siglo XXI. 
Guba, Egon. 1990. The paradigm dialog. California: Sage Publication Inc.

Gudynas, Eduardo. 2012. Debates sobre el desarrollo y sus alternativas en América Latina: una breve guía heterodoxa. En Más allá del desarrollo. Grupo Permanente de Trabajo sobre Alternativas al Desarrollo. Quito: Fundación Rosa Luxemburgo/Ediciones Abya Yala.

Guizar, Francisco. 2005. Estrategias de apropiación territorial y de construcción de hegemonía en un contexto intergrupal: el caso de San Lucas de Jalpa, El Mezquital, Durango. Revista Relaciones (101): 83-121.

Gunderson, Lance y Crawdord Holling. 2002. Panarchy; understanding transformations in systems of humans and nature. Washington: Island Press.

Haesbaert, Rogerio. 2004. O mito da desterritorializacao: do "fim dos territorios" a multiterritorialidade. Río de Janeiro: Bertrand Brasil.

Harvey, David. 2007a. Espacios del capital hacia una geografía crítica. Madrid: Ediciones Akal.

Harvey, David. 2007b. Notas hacia una teoría del desarrollo geográfico desigual. Apuntes de geografía y ciencias sociales. Universidad de Buenos Aires.

Harvey, David. 2005. El "nuevo” imperialismo: acumulación por desposesión. Socialist Register 2004 (Enero): 99-129.

Harvey, David. 2000. Espacios de esperanza. Madrid, Ediciones Akal. Hiernaux, Daniel y Alicia Lindón. 2012. Geografías de lo imaginario. Barcelona-México: Anthropos, UAM-I.

Hiernaux, Daniel. 1999. Los fundamentos territoriales del liberalismo contemporáneo. En El pensamiento único. Fundamentos y política económica, compilado por Elvira Concheiro, 149-165. México: UAM, Instituto de Investigaciones Económicas-UNAM. 
Holling, C. S. 2001. Understanding the complexity of economic, ecological, and social systems. Ecosystems 4 (5): 390-405.

Holling, C. S., L. H. Gunderson y D. Ludwig. 2002. In quest of a theory of adaptive change. En Panarchy: understanding transformations in human and natural systems, editado por L. H Gunderson y C. S. Holling, 3-24. Washington: Island Press.

Jiménez, Marcela. 2013. Resiliencia y adaptabilidad del sistema chinampero de Xochimilco. Tesis de licenciatura en geografía, UNAM.

Kropotkin, Piotr. 1902. El apoyo mutuo. http://www.cgt.info/descargas/SalaLectura/kropotkin-apoyo-mutuo.pdf (30 de septiembre de 2013).

Llanos, Luis. 2010. El concepto del territorio y la investigación en ciencias sociales. Agricultura, Sociedad y Desarrollo VII (3): 207-220.

Lang, Miriam y Dubia Mokrani. 2012. Más allá del desarrollo. Grupo Permanente de Trabajo sobre Alternativas al Desarrollo. Quito: Fundación Rosa Luxemburgo. Ediciones Abya Yala.

Leff, Enrique. 2003a. Racionalidad ambiental y diálogo de saberes: sentidos y senderos de un futuro sustentable. Desenvolvimento e Meio Ambiente (7): 13-40.

Leff, Enrique. 2003b. La ecología política en América Latina. Un campo en construcción. Panamá: Grupo de Ecología Política de CLACSO.

Leff, Enrique, Arturo Argueta, Eckart Boege y Carlos W. Porto. 2002. Globalización, interculturalidad y diálogo de saberes. http:/ /www. revistafuturos.info/futuros_9/ds_al_2.htm. (9 de septiembre de 2012).

Lyon, Arthur. 1996. Medir lo inconmensurable. Nuestro Planeta 8 (1): 29-33. 
Márquez, Conrado. 2002. Apropiación territorial, gestión de recursos comunes y agricultura campesina en la selva Lacandona, Chiapas. Pueblos y Fronteras (3): 26-51.

Mallon, Florencia. 1995. Campesinado y nación. La construcción de México y Perú poscoloniales. México: Centro de Investigación y Estudios Superiores en Antropología Social, El Colegio de Michoacán, El Colegio de San Luis Potosí.

Masera, Omar, Martha Astier y Santiago López-Ridaura. 1999. Definición y atributos sistémicos de la agricultura sustentable. Los conceptos de sustentabilidad y desarrollo sustentable. En Sustentabilidad y manejo de recursos naturales. El marco de evaluación mesmis. México: Mundi Prensa, Grupo Interdisciplinario de Tecnología Rural Apropiada, Instituto de Ecología.

Merino, Leticia. 1999. La gestión colectiva de los recursos forestales. http://www.era-mx.org/publicaciones/gestion.html (30 de septiembre de 2013).

Monroy, Francisco. 2004. La transformación del espacio en la era global. Convergencia 11 (36): 131-158.

Ortiz, Renato. 2005. Mundialización: saberes y creencias. Barcelona: Gedisa.

Ostrom, Elinor. 2000. El gobierno de los bienes comunes: la evolución de las instituciones de acción colectiva. México: FCE.

Palacios, Juan José. 1983. El concepto de región: la dimensión espacial de los procesos sociales. Revista Interamericana de Planificación (66): 56-68.

PNUD. 1995. Informe sobre desarrollo humano 1994. http://hdr. undp.org/en/media/hdr_1994_es_cap1.pdf (4 de noviembre de 2013).

Pretty, Jules. 1995. Sustainable agriculture. En Regenerating agriculture. Politics and practice for sustainability end self-reliance, 2-25. Londres: Earthscan Publications Ltd. 
Puydt, Paul. 1860. Panarchy. http://www.panarchy.org/depuydt/1860.eng.html (17 de marzo de 2014).

Raffestin, Claude. 1993. Por uma geografia do poder. Sao Paulo: Editora Ática.

Ramírez, Blanca. 2003. Modernidad, posmodernidad, globalización y territorio. México: UAM, Miguel Ángel Porrúa.

Reclus, Elisée. 1905. El hombre y la Tierra. México: FCE.

Resilience Alliance. 2013. Resilience Alliance home page. http:// www.resalliance.org (17 de marzo de 2014).

Roling, N. G. y Annemarie Wagemakers. 2000. A new practice: facilitating sustainable agriculture. Facilitating sustainable agriculture. En Participatory learning and adaptive management in times of environmental uncertainty, 3-21. Cambridge: Cambridge University Press.

Santos, Milton. 2000. La naturaleza del espacio. Técnica y tiempo, razón y emoción. Barcelona: Ariel.

Serrano, Jorge. 1999. Desarrollo regional y urbano en México a finales del siglo xx: una agenda de temas pendientes. En La globalización y las regiones en México (III), coordinado por Jorge Serrano. México: Asociación Mexicana de Ciencias para el Desarrollo Regional, UNAM, Universidad Autónoma del Estado de México.

Schneider, Sergio e Iván G. Peyré. 2006. Territorio y enfoque territorial: de las referencias cognitivas a los aportes aplicados al análisis de los procesos sociales rurales. En Desarrollo rural. Organizaciones, instituciones y territorio. Buenos Aires: Ciccus.

Sloterdijk, Peter. 2007. En el mundo interior del capital. Para una teoría filosófica de la globalización. Madrid: Siruela.

United Nations. 1987. Report of the World Commission on Environment and Development. Our common future. Nueva York: Naciones Unidas. 
Wallerstein, Immanuel. 2006. Análisis de sistemas-mundo, una introducción. México: Siglo XXI.

Watzlawick, Paul. 1981. ¿Es real la realidad?: confusión, desinformación, comunicación. Barcelona: Editorial Herder S.A.

Weeks, Brian, Marco A. Rodríguez y J. H. Blakeslee. 2004. Panarchy: complexity and regime change in human societies. California: Institute Complex Systems.

Woodhill, James y N. C. Roling. 2000. The second wind of the eagle: the human dimension in learning our way to more sustainable futures. En Facilitating sustainable agriculture: participatory learning and adaptive management in times of environmental uncertainty, 46-71. Cambridge: Cambridge University Press.

Zibechi, Raúl. 2003. Los movimientos sociales latinoamericanos: tendencias y desafíos. Revista del Observatorio Social de América Latina (9) 185-188.

Zúñiga, Gerardo. 1998. Los procesos de constitución de territorios indígenas en América Latina. Nueva Sociedad (153): 141-155. 
